\title{
The LncRNA NEAT1 Accelerates Lung Adenocarcinoma Deterioration and Binds to Mir-193a-3p as a Competitive Endogenous RNA
}

\author{
Dan-dan Xionga Zu-yun Lia Lu Liang ${ }^{a} \quad$ Rong-quan He ${ }^{b} \quad$ Fu-chao Mab \\ Dian-zhong Luo Xiao-hua Hu ${ }^{b}$ Gang Chen ${ }^{a}$ \\ aDepartment of Pathology, First Affiliated Hospital of Guangxi Medical University, Nanning, \\ 'Department of Medical Oncology, First Affiliated Hospital of Guangxi Medical University, Nanning, \\ China
}

\section{Key Words}

Neat1 $・$ LUAD $\cdot$ MiR-193a-3p • Biological function $•$ CeRNA

\begin{abstract}
Background/Aims: Long noncoding RNAs (IncRNAs) contribute to the development of multiple malignant tumors. Here, we focused on the biological function and underlying molecular mechanism of an IncRNA, nuclear-enriched abundant transcript 1 (NEAT1), in lung adenocarcinoma (LUAD). Methods: In vitro experiments were conducted to determine the biological effects of NEAT1 in LUAD cells. A luciferase activity reporter assay was performed to corroborate the interaction between NEAT1 and miR-193a-3p. Data from Gene Expression Omnibus (GEO), Oncomine, The Cancer Genome Atlas (TCGA), and our in-house reverse transcription quantitative PCR (RT-qPCR) were combined to examine the expression of NEAT1 and miR-193a-3p in LUAD. To further explore the regulatory mechanism of NEAT1, we searched for putative target genes of miR-193a-3p from 12 online prediction databases and determined genes positively correlated with NEAT1 as candidate targets. Furthermore, we analyzed the expression of these selected genes using data from TCGA. Results: In vitro experiments showed that knockdown of NEAT1 in LUAD cells markedly restrained cell proliferation, invasion, and migration and stimulated cell apoptosis. The dual-luciferase reporter assay demonstrated that miR-193a-3p directly targeted NEAT1 at its 3'-UTR. We then detected NEAT1 and miR-193a$3 p$ in LUAD cells and normal lung epithelial cells and discovered high expression of NEAT1 and low expression of miR-193a-3p in LUAD cell lines. Simultaneously, the pooled results from the GEO, Oncomine, TCGA, and in-house RT-qPCR showed that the NEAT1 expression increased while the miR-193a-3p expression decreased in LUAD tissues versus normal lung tissues. Furthermore, the USF1 gene was not only upregulated in LUAD, but also positively correlated with NEAT1, suggesting that NEAT1 may function as a ceRNA to sponge miR-193aD.-d. Xiong and Z.-y. Li contributed equally to this work.

\footnotetext{
Xiao-hua Hu

Department of Medical Oncology and Pathology, First Affiliated Hospital of Guangxi Medical University and Gang Chen

6 Shuangyong Road, Nanning 530021, Guangxi Zhuang Autonomous Region (China)

Tel. +8615277192143, E-Mail gxmuhxh@163.com; chengang@gxmu.edu.cn
} 
$3 p$ and abrogate the inhibitory effect of miR-193a-3p on USF1. Conclusions: Our findings indicate that NEAT1 plays important roles in the occurrence and progression of LUAD. It may exert its role by acting as a ceRNA to regulate miR-193a-3p.

(C) 2018 The Author(s)

Published by S. Karger AG, Basel

\section{Introduction}

Lung cancer is the main cause of oncogenic mortality in both men and women worldwide $[1,2]$. Non-small cell lung carcinoma (NSCLC), the most prevalent form of lung cancer, includes three histopathological subtypes: adenocarcinoma, squamous cell carcinoma, and large cell carcinoma [3]. Lung adenocarcinoma (LUAD) is the most common variant, accounting for nearly $40 \%$ of lung cancer cases [3, 4]. Despite improved understanding of oncogenic drivers, the potential molecular mechanism of LUAD remains unclear. Thus, investigation of the possible mechanism of LUAD pathogenesis is an urgent and challenging task.

Increasing numbers of studies have concentrated on long noncoding RNAs (lncRNAs), which may be cell-type specific RNAs that contribute to tumor onset and progression via multiple ways, including cell cycle regulation, transcriptional/post-transcriptional regulation, and DNA methylation [5-8]. Nuclear-enriched abundant transcript 1 (NEAT1), a structural component of paraspeckle, plays critical roles in several malignancies, including lung cancer. Our research group has previously examined the expression of NEAT1 in NSCLC via reverse transcription quantitative PCR (RT-qPCR) [9]. The results indicated that NEAT1 was upregulated in NSCLC compared with adjacent non-tumorous tissues, with overexpressed NEAT1 strongly associated with several malignant clinical phenotypes, such as lymphatic metastasis, TNM stage, and vascular invasion. A study by Sun et al [10]. showed that NEAT1 played key roles in NSCLC by promoting the Wnt/ $\beta$-catenin pathway. Accumulating studies have reported that lncRNAs, including NEAT1, exert roles in tumors by functioning as miRNA sponges or competing endogenous RNAs (ceRNAs) to interact with miRNAs. In NSCLC, NEAT1 has been demonstrated to competitively bind to miRNAs, such as miR-181a-5p and miR-98, and subsequently upregulate their target genes $[11,12]$. Sun et al [13]. discovered that NEAT1 accelerated NSCLC development by acting as a ceRNA for miR-377-3p and antagonizing the inhibition of miR-377-3p on its target gene E2F3, a critical oncogene in NSCLC carcinogenesis. Similarly, the impact of NEAT1 on the miR-377-3p/E2F3 axis in NSCLC was recapitulated in a study conducted by Zhang et al [14].. However, the understanding of the underlying role and functional mechanism of NEAT1 in LUAD is still limited. We therefore carried out this study to elucidate the possible regulatory mechanism of NEAT1 in LUAD.

In the present study, we first performed in vitro experiments to investigate the biological function of NEAT1 in LUAD. A luciferase activity assay was then conducted to verify the interaction between NEAT1 and miR-193a-3p. Microarray and RNA-seq data were mined to validate the expression of NEAT1 and miR-193a-3p in LUAD. Furthermore, the potential targets of miR-193a-3p were predicted by online tools, and bioinformatics analysis was subsequently performed to explore the active mechanism of NEAT1 in LUAD.

\section{Materials and Methods}

\section{Cell culture and transfection}

Four LUAD cell lines (H1299, A549, HCC827, and Calu3), one human renal epithelial cell line (293T), and one normal lung epithelial cell line (BEAS-2B) were purchased from ScienCell Research Laboratories (Carlsbad, CA) and cultured in RPMI-1640 (Invitrogen, Carlsbad, CA) medium supplemented with 10\% fetal bovine serum (Biological Industries, Beit Haemek, Israel) and 1\% penicillin/streptomycin (Invitrogen). Cells were transfected with mock control, blank control, scrambled siRNAs, and NEAT1 siRNAs for in vitro experiments according to the manufacturer's instructions. After 48 hours, the transfection efficiency was verified via RT-qPCR. 


\section{Cellular Physiology Cell Physiol Biochem 2018:48:905-918 \begin{tabular}{l|l} 
and Biochemistry Published online: July 23, 2018 & $\begin{array}{l}\text { (c) } 2018 \text { The Author(s). Published by S. Karger AG, Basel } \\
\text { www.karger.com/cpb }\end{array}$ \\
\hline
\end{tabular}}

Xiong et al.: NEAT1 Promotes Lung Adenocarcinoma Deterioration

\section{Expression of NEAT1 and miR-193a-3p by RT-qPCR}

Cellular RNA was isolated using TRNzol reagent (TIANGEN, Beijing, China) and converted to cDNA using TransScript One-Step gDNA Removal and cDNA Synthesis SuperMix kits (TransGen Biotech, Beijing, China). Subsequently, RT-qPCR was carried out with a CFX96TM Touch PCR system (BioRad Laboratories, Hercules, CA). NEAT1 primer sequences (forward 5'-TTACCAGCTTCCTCCTGGTG-3', reverse 5'-TCTGCTGCGTATGCAAGTCT-3') and the miR-193a-3p forward primer sequence (5'-TAACTGGCCTACAAAGTCCCA-3') were synthesized by Sangon Biotech (Shanghai, China). HS-ACTB (Sangon Biotech) served as the endogenous control. Relative NEAT1 and miR-193a-3p abundances were determined with the $2^{-\Delta \mathrm{CT}}$ method [15].

\section{Biological effects of NEAT1 on LUAD cells in cell functional experiments}

We selected cell lines for further in vitro experiments based on the aforementioned RT-qPCR results. Cell viability and proliferation were detected with fluorometric detection of resorufin (CellTiter-Blue Cell Viability Assay, G8080, Promega, Madison, WI) and colorimetric tetrazolium (MTS) assay (CellTiter96 AQueous One Solution Cell Proliferation Assay G3580, Promega), respectively. Cell apoptosis was examined with Hoechst 33342 and propidium iodide (PI; Sigma-Aldrich, St. Louis, MO) double-fluorescent chromatin staining and the Apo-ONE® Homogeneous Caspase-3/7 Assay (G7790, Promega). Two reviewers independently counted the viable and apoptotic cells in 10 fields of each well under a $200 \times$ magnification microscope after Hoechst 33342/PI double-fluorescent chromatin staining at 96 hours. A wound-healing assay was conducted to monitor cell migration. Photographs of the wound were obtained at $0,12,24$, and 48 hours to observe cell migration and calculate the wound width. The wound widths at 12,24 , and 48 hours were compared with the wound width of the blank control at 0 hours. A Transwell assay was performed to detect the effects of NEAT1 on LUAD cell invasion. Cells were independently counted in five random fields under a microscope by two investigators. All in vitro experiments were conducted as reported in preceding studies [16-19].

\section{Validation of NEAT1 and miR-193a-3p binding based on luciferase activity}

To corroborate whether NEAT1 directly binds to miR-193a-3p, a human renal cell line 293T was chosen for the luciferase activity assay due to its high transfection efficiency. The wild-type (WT) NEAT1 3'UTR binding site of miR-193a-3p was subcloned into pGL3 Basic vector (Promega). Similarly, the WT TRAF6 3'-UTR binding site of miR-146 was also cloned into pGL3 Basic vector (Promega) as a positive control [20, 21]. WT NEAT1-3'-UTR or WT TRAF6-3'-UTR vector and miRNA mimic or negative control (NC) plasmid were then co-transfected into 293T cells. After 48 hours, the luciferase activity was determined with the Dual-Luciferase ${ }^{\circledR}$ Reporter Assay System (Promega) in accordance with the manufacturer's protocols.

\section{Statistical analysis}

All experiments were performed in triplicate, and continuous variable results are presented as the mean \pm standard deviation (SD). A Student's t test or one-way analysis of variance was used for comparisons between groups. All statistical analyses were carried out using SPSS 22.0 (IBM, Armonk, NY) and GraphPad Prism v5.0 (GraphPad Software, Inc., La Jolla, CA). P<0.05 indicated statistical significance.

\section{Clinical roles of NEAT1 and miR-193a-3p based on meta-analyses}

To investigate the expression and correlation of NEAT1 and miR-193a-3p in LUAD, we performed a meta-analysis based on data from Gene Expression Omnibus (GEO; http://www.ncbi.nlm.nih.gov/geo/), Oncomine (https://www.oncomine.org/resource/main.html), The Cancer Genome Atlas (TCGA; https:// cancergenome.nih.gov/), and our in-house RT-qPCR. The following search strategies were used to retrieve eligible datasets from GEO: (lung OR pulmonary OR LC OR LUAD) AND (miRNA OR microRNA OR miR OR "non-coding RNA" OR ncRNA OR IncRNA OR "noncoding RNA" OR "non coding RNA"). The inclusion criteria were as follows: (i) available expression data of NEAT1 and miR-193a-3p; (ii) a sample size of each dataset in cancer and non-cancerous groups exceeding 3; (iii) human species; and (iv) expression data in the miRNArelated dataset for mature miR-193a-3p.

The following characteristics of each included dataset were collected: first author and published year, region, data source, platform, sample size, and expression level of NEAT1 or miR-193a-3p. 


\section{Cellular Physiology Cell Physiol Biochem 2018;48:905-918 \begin{tabular}{l|l} 
DOI: 10.1159/000491958 & $\begin{array}{l}\text { O 2018 The Author(s). Published by S. Karger AG, Basel } \\
\text { www.karger.com/cpb }\end{array}$
\end{tabular}}

The pooled standard mean difference (SMD) and corresponding 95\% confidence intervals (CIs) were calculated to assess the overall expression levels of NEAT1 and miR-193a-3p in LUAD. A summary receiver operating characteristic (sROC) curve was generated to illustrate the ability of NEAT1 and miR-193a$3 p$ expression to discriminate LUAD patients from normal controls. The heterogeneity among included studies was evaluated by the $\chi 2$-based $\mathrm{Q}$ test and $\mathrm{I}^{2}$ statistic. A random-effects model was used if there was heterogeneity $\left(\mathrm{I}^{2}>50 \%\right.$ or $\left.\mathrm{P}<0.05\right)$. Otherwise, a fixed-effects model would be chosen [22]. Sensitivity analysis was implemented to assess the stability of the pooled SMD. Begg's funnel plot and Egger's test were used to ascertain the possible publication bias. $\mathrm{P}>0.05$ favored no publication bias.

\section{Identification of potential targets of miR-193a-3p based on bioinformatics analysis}

Potential targets of miR-193a-3p were forecasted in miRWalk 2.0 (http://zmf.umm.uni-heidelberg.de/ apps/zmf/mirwalk2/); this database contains 12 prediction algorithms (TargetScan, RNAhybrid, RNA22, PITA, Pictar2, miRWalk, Microt4, miRNAMap, miRDB, miRBridge, miRanda, and miRmap). Genes appearing in at least 8 algorithms were considered to be putative targets of miR-193a-3p. Additionally, genes positively correlated with NEAT1 were obtained from TCGA. The intersection between the predicted targets and positively correlated genes was determined. Then, we evaluated the expression levels of the overlapping genes with data from TCGA.

\section{Results}

Knockdown of NEAT1 inhibits LUAD cell proliferation and triggers cell apoptosis in vitro

To determine the biological role of NEAT1 in LUAD cell proliferation and apoptosis, we established NEAT1-knockdown cell lines (A549 and Calu3) using siRNAs, with knockdown efficiencies from $75 \%$ to $92 \%$. Fluorometric detection of resorufin and an MTS assay showed that silencing of NEAT1 in vitro markedly arrested cell viability and proliferation in both A549 and Calu3 cells (Fig. 1). In addition, the effects of NEAT1 on LUAD cell apoptosis were detected by Hoechst 33342/PI double-fluorescent chromatin staining and caspase-3/7 assay. The results indicated that the apoptosis and caspase-3/7 activity of A549 and Calu3 cell lines following NEAT1 knockdown were clearly increased (Fig. 2A-2D). Simultaneously, we observed viable and apoptotic cells under a microscope after Hoechst 33342/PI staining at 96 hours. The results suggested that NEAT1 siRNAs apparently stimulated apoptosis and restrained viability of A549 and Calu3 cell lines (Fig. 2E).

\section{Knockdown of NEAT1 reduces LUAD cell invasion and migration in vitro}

Next, we silenced NEAT1 in H1299 cells and subsequently examined the effect of NEAT1 on cell invasion and migration by wound-healing and Transwell assays. Our results showed that the invasion and metastasis of NEAT1-knockdown cells were markedly decreased compared with control cells (Figs. 3, 4), suggesting that NEAT1 siRNAs impeded LUAD cell invasive and metastatic ability in vitro.

Fig. 1. NEAT1 siRNAs restrain the viability and proliferation of A549 and Calu3 cells. Mock control, blank control, scrambled siRNAs, and NEAT1 siRNAs were transfected into A549 and Calu3 cells for 0, 24, 48, 72, and 96 hours. (A) Effects of NEAT1 siRNAs on A549 cell viability evaluated by CellTiter-Blue Cell Viability Assay. (B) Effects of NEAT1 siRNAs on Calu3 cell viability evaluated by CellTiter-Blue Cell Viability Assay. (C) Effects of NEAT1 siRNAs on A549 cell proliferation evaluated by MTS assay. (D) Effects of NEAT1 siRNAs on Calu3 cell proliferation by evaluated by MTS assay. ${ }^{*} \mathrm{P}<0.05 ;{ }^{* *} \mathrm{P}<0.01$; $* * * \mathrm{P}<0.001$.

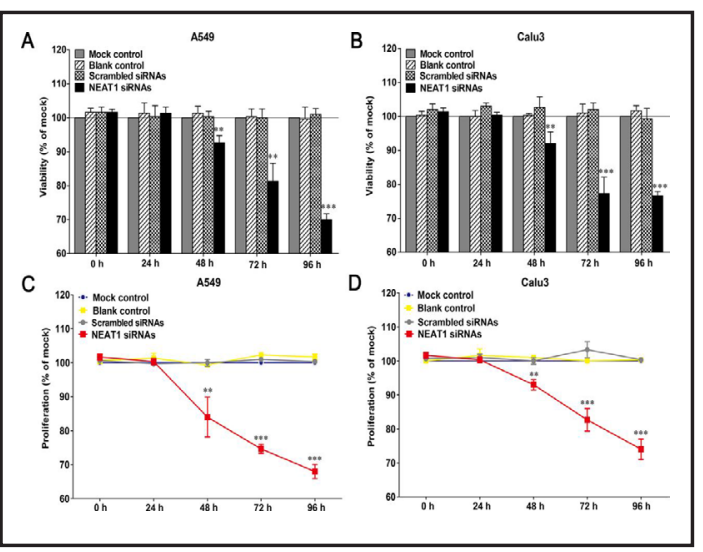


NEAT1 directly binds to miR-193a-3p in LUAD cells

Multiple studies have demonstrated that IncRNAs could serve as ceRNAs or molecular sponges of miRNAs in multiple malignancies. To determine whether NEAT1 exerts a similar mechanism in LUAD cells, we screened miRNAs containing putative binding sites in NEAT1 with microRNA.org (http://34.236.212.39/microrna/home.do). miR-193a-3p was found to harbor multiple binding sites in NEAT1 (Fig. 5A). Then, we examined the expression of NEAT1 and miR-193a-3p in LUAD cell lines (A549, H1299, and HCC827) and a normal

Fig. 2. NEAT1 siRNAs induce apoptosis in A549 and Calu 3 cells. (A) Effects of NEAT1 siRNAs on A549 cell apoptosis evaluated by fluorescence microscopy after Hoechst 33342/PI staining. (B) Effects of NEAT1 siRNAs on Calu3 cell apoptosis evaluated by fluorescence microscopy after Hoechst 33342/ PI staining. (C) Effects of NEAT1 siRNAs on A549 cell apoptosis per caspase $3 / 7$ activity evaluated by the Apo-ONE® Homogeneous Caspase-3/7 Assay. (D) Effects of NEAT1 siRNAs on Calu3 cell apoptosis per caspase $3 / 7$ activity evaluated by the Apo-ONE® Homogeneous Caspase-3/7 Assay. ${ }^{*} \mathrm{P}<0.05$; ${ }^{* *} \mathrm{P}<0.01 ;{ }^{* * *} \mathrm{P}<0.001$. (E) NEAT1 siRNAs inhibit the viability and stimulated apoptosis of A549 and Calu3 cells. Hoechst 33342-positive/PI-negative (white arrow): viable cells; Hoechst 33342-positive/PI-negative with blue fragmentation in the cells (red arrow): early apoptotic cells; Hoechst 33342-positive/PIpositive with red fragmentation in the cells (pink

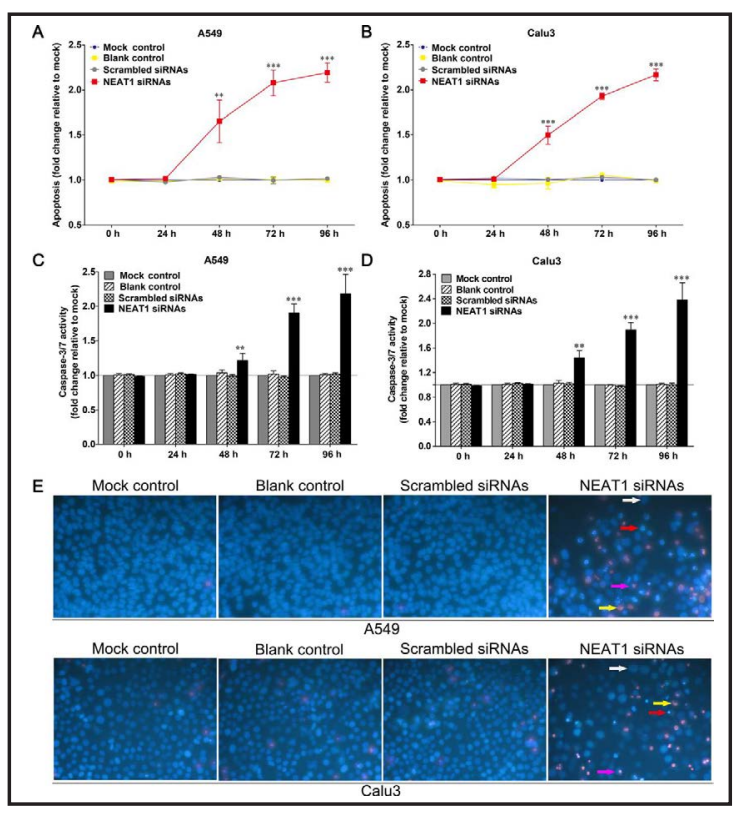
arrow): late apoptotic cells; PI-positive with debris signals (yellow arrow): necrotic cells.

Fig. 3. NEAT1 siRNAs inhibit the migration of H1299 cells. H1299 cells were transfected with blank control, scrambled siRNAs, and NEAT1 siRNAs for $0,12,24$, and 48 hours. (A) Cell migration was examined in wound-healing assays. (B) The wound width was calculated. The cell migration and would width are shown by red arrows.

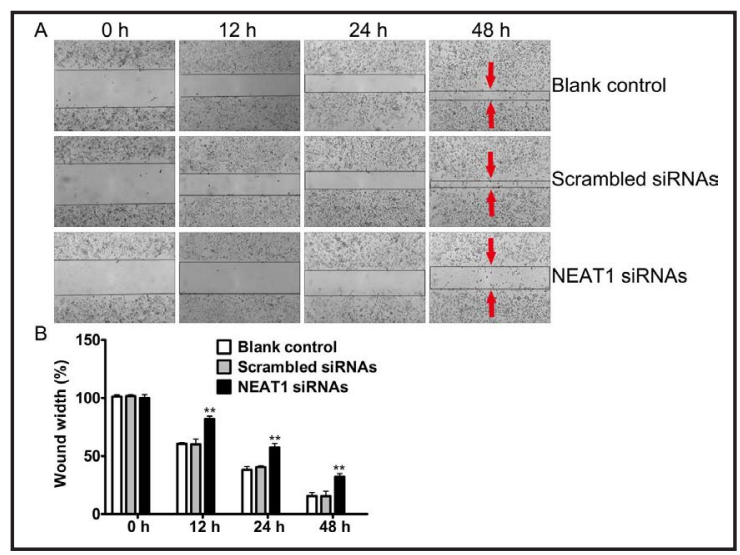

Fig. 4. NEAT1 siRNAs inhibit the invasion of H1299 cells. H1299 cells were transfected with a blank control, scrambled siRNA, and NEAT1 siRNA for 48 hours. (A) Cell invasion was examined with Transwell assays. (B) The number of invasive cells was counted. The invasive cells are shown by red arrows.

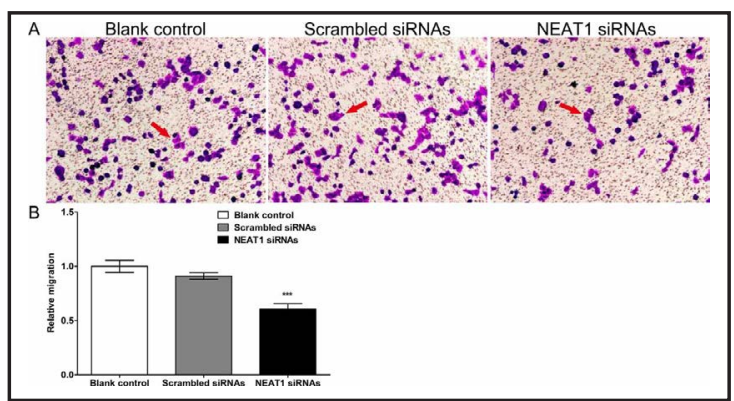


Fig. 5. Relationship between NEAT1 and miR-193a-3p. (A) Predicted miR-193a-3p binding sites in NEAT1. (B) Expression levels of NEAT and miR-193a-3p in lung adenocarcinoma (LUAD) cell lines (A459, H1299, and HCC87) and a normal lung epithelial cell line (BEAS-2B). (C) Dual-luciferase reporter assay confirmed that NEAT1 directly bound to miR193a-3p in LUAD cells.

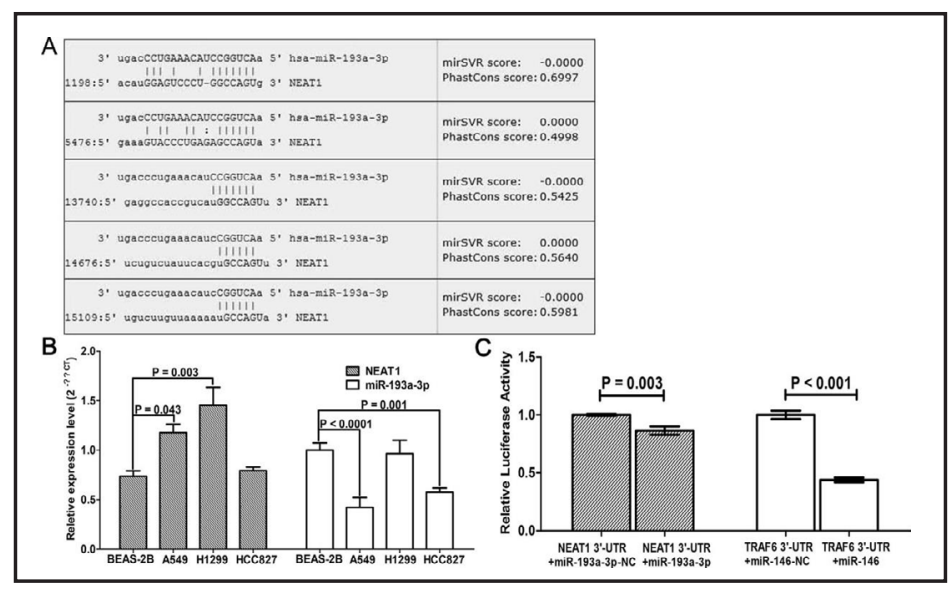

epithelial cell line (BEAS2B). As shown in Fig. 5B, NEAT1 was significantly upregulated in A549 and H1299 LUAD cells compared with BEAS-2B cells. In addition, miR-193a-3p was clearly downregulated in A549 and HCC827 LUAD cells compared with BEAS$2 \mathrm{~B}$ cells. Furthermore, to determine whether NEAT1 directly binds to miR-193a$3 p$, we carried out a dualluciferase reporter assay. Our results demonstrated that miR-193a-3p decreased the luciferase activity of the NEAT1 3'-UTR, indicating direct binding between NEAT1 and miR-193a-3p (Fig. 5C).

\section{NEAT1 is upregulated while miR-193a-3p is downregulated in LUAD tissues based on meta- analysis \\ To further elucidate}

Table 1. Main information on the 11 included datasets for NEAT1. T: tumor; N: normal; SD: standard deviation; TCGA: The Cancer Genome Atlas; RT-qPCR: reverse transcription quantitative PCR

\begin{tabular}{|c|c|c|c|c|c|c|}
\hline \multirow[t]{2}{*}{$\begin{array}{l}\text { First author } \\
\text { (year) }\end{array}$} & \multirow[t]{2}{*}{ Region } & \multirow[t]{2}{*}{ Data source } & \multirow[t]{2}{*}{ Platform } & \multirow{2}{*}{$\begin{array}{l}\text { Sample size } \\
(\mathrm{T} / \mathrm{N})\end{array}$} & \multicolumn{2}{|c|}{$\begin{array}{l}\text { NEAT expression } \\
\text { (mean } \pm \mathrm{SD})\end{array}$} \\
\hline & & & & & $\mathrm{T}$ & \\
\hline Su LJ (2007) & China & GSE7670 & GPL96 & $27 / 28$ & $8.26 \pm 0.87$ & $7.82 \pm 0.86$ \\
\hline $\begin{array}{l}\text { Landi MT } \\
\text { (2008) }\end{array}$ & USA & GSE10072 & GPL96 & $58 / 49$ & $7.03 \pm 0.71$ & $6.84 \pm 0.78$ \\
\hline Hou J (2010) & Netherlands & GSE19188 & GP570 & $43 / 63$ & $1.44 \pm 1.01$ & $1.24 \pm 0.79$ \\
\hline $\begin{array}{l}\text { Kohno T } \\
(2011)\end{array}$ & Japan & GSE31210 & GPL570 & $226 / 20$ & $6.29 \pm 0.49$ & $5.96 \pm 0.30$ \\
\hline Li-Jen S (2013) & China & GSE27262 & GPL570 & $25 / 25$ & $1.10 \pm 0.29$ & $0.92 \pm 0.08$ \\
\hline Feng L (2014) & China & GSE43767 & GPL6480 & $69 / 34$ & $11.59 \pm 1.47$ & $9.96 \pm 2.04$ \\
\hline $\begin{array}{l}\text { Garber ME } \\
\text { (2001) }\end{array}$ & USA & Oncomine & None & $30 / 6$ & $2.33 \pm 1.14$ & $1.32 \pm 0.53$ \\
\hline $\begin{array}{l}\text { Beer DG } \\
(2002)\end{array}$ & USA & Oncomine & None & $86 / 10$ & $0.23 \pm 0.31$ & $0.08 \pm 0.07$ \\
\hline $\begin{array}{l}\text { Okayama H } \\
(2012)\end{array}$ & Japan & Oncomine & None & $226 / 20$ & $2.40 \pm 0.96$ & $2.20 \pm 0.51$ \\
\hline TCGA (2017) & USA & TCGA & None & $535 / 59$ & $14.55 \pm 1.58$ & $13.84 \pm 1.20$ \\
\hline Pan LJ (2015) & China & $\begin{array}{l}\text { In-house RT- } \\
\text { qPCR }\end{array}$ & None & 101/101 & $6.79 \pm 3.64$ & $4.92 \pm 3.12$ \\
\hline
\end{tabular}

Table 2. Main information on the 10 included datasets for miR-193a3p. T: tumor; N: normal; SD: standard deviation; RT-qPCR: reverse transcription quantitative PCR. T: tumor; N: normal; SD: standard deviation; RT-qPCR: reverse transcription quantitative PCR

\begin{tabular}{lcccccc}
\hline $\begin{array}{l}\text { First author } \\
\text { (year) }\end{array}$ & Region & Data source & Platform & $\begin{array}{c}\text { Sample size } \\
\text { (T/N) }\end{array}$ & \multicolumn{2}{c}{$\begin{array}{c}\text { MiR-193a-3p expression } \\
\text { (mean } \pm \text { SD) }\end{array}$} \\
\hline Guled M (2011) & Finland & GSE25508 & GPL7731 & $5 / 5$ & $6.72 \pm 0.75$ & $6.43 \pm 0.50$ \\
Ma L (2012) & China & GSE29248 & GPL8179 & $3 / 3$ & $9.73 \pm 0.37$ & $9.33 \pm 0.32$ \\
Ohba T (2013) & Japan & GSE19945 & GPL9948 & $4 / 8$ & $2.78 \pm 1.07$ & $3.59 \pm 0.55$ \\
van Jaarsveld & Netherlands & GSE47525 & GPL17222 & $6 / 8$ & $4.02 \pm 0.44$ & $3.89 \pm 0.97$ \\
MT (2013) & & & & & & \\
Bjaanaes MM & Norway & GSE48414 & GPL16770 & $153 / 19$ & $1.09 \pm 0.60$ & $1.13 \pm 0.22$ \\
(2014) & Japan & GSE51853 & GPL7341 & $76 / 5$ & $0.62 \pm 0.48$ & $0.95 \pm 0.17$ \\
Arima C (2014) & USA & GSE63805 & GPL18410 & $16 / 14$ & $0.98 \pm 0.92$ & $1.91 \pm 1.27$ \\
Robles AI (2015) & China & GSE74190 & GPL19622 & $36 / 44$ & $2.72 \pm 1.21$ & $3.14 \pm 0.93$ \\
Jin Y (2015) & Japan & GSE77380 & GPL16170 & $3 / 6$ & $4.97 \pm 0.67$ & $5.94 \pm 0.64$ \\
Yoshimoto T & I2016) & In-house RT- & None & $101 / 101$ & $4.47 \pm 2.82$ & $7.19 \pm 2.97$ \\
Ren FH (2015) & China & qPCR & & & & \\
\hline
\end{tabular}

the expression of and correlation between NEAT1 and miR-193a-3p in LUAD, we obtained data from GEO, Oncomine, TCGA, and RT-qPCR on-file to conduct comprehensive analyses. A total of 11 records with 1405 LUAD samples and 415 non-cancerous samples were included for NEAT1 and 10 records with 402 LUAD samples and 213 non-cancerous samples for miR-193a-3p. The basic information on these studies is included in Table 1 and Table 2. The expression of NEAT1 and miR-193a-3p in each dataset is presented as a box-scatter plot and receiver operating characteristic (ROC) curve (Figs. 6-9). Due to the divergences in individual studies, we then combined all of the included datasets. The pooled results showed that the expression level of NEAT1 was higher in LUAD tissues than in normal lung tissues (SMD $=0.44,95 \% \mathrm{CI}=0.32$ - 
Fig. 6. Box-scatter plots for NEAT1 expression in LUAD. (A) GES7670. (B) GSE10072. (C) GSE19188. (D) GSE31210. (E) GSE27262. (F) GSE43767. (G) Oncomine: Garber ME. (H) Oncomine: Beer DG. (I) Oncomine: Okayama H. (J) TCGA. (K) In-house RTqPCR.

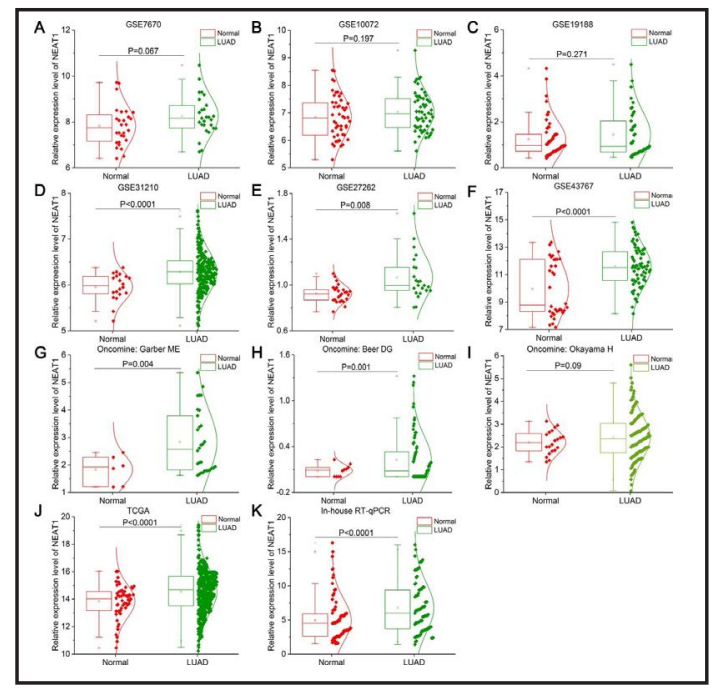

Fig. 7. ROC curves for NEAT1 expression in LUAD. (A) GES7670. (B) GSE10072. (C) GSE19188. (D) GSE31210. (E) GSE27262. (F) GSE43767. (G) Oncomine: Garber ME. (H) Oncomine: Beer DG. (I) Oncomine: Okayama H. (J) TCGA. (K) In-house RTqPCR.

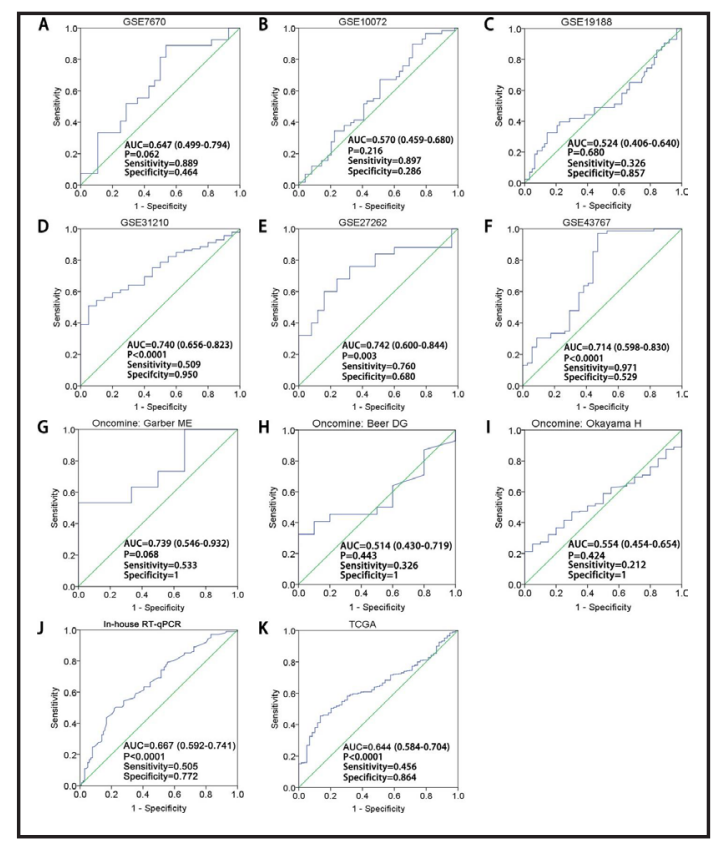

Fig. 8. Box-scatter plots for miR-193a-3p expression in LUAD. (A) GES25508. (B) GSE29248. (C) GSE19945. (D) GSE47525. (E) GSE48414. (F) GSE51853. (G) GSE63805. (H) GSE74190. (I) GSE77380. (J) In-house RT-qPCR.

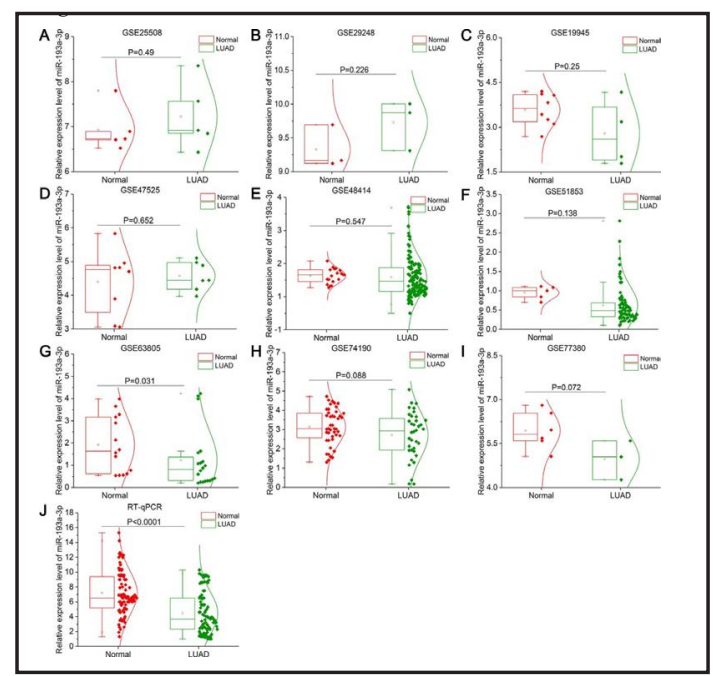


Fig. 9. ROC curves for miR-193a-3p expression in LUAD. (A) GES25508. (B) GSE29248. (C) GSE19945. (D) GSE47525. (E) GSE48414. (F) GSE51853. (G) GSE63805. (H) GSE74190. (I) GSE77380. (J) In-house RT-qPCR.

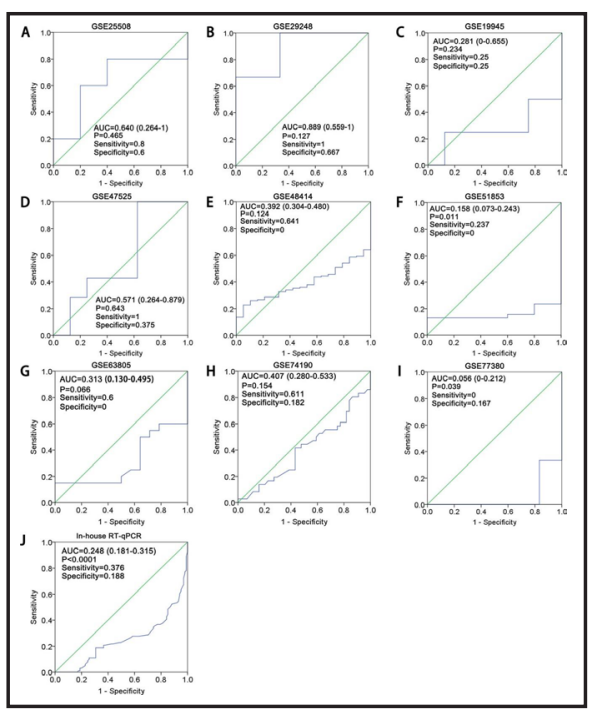

Fig. 10. Expression levels of NEAT1 and miR-193a-3p in LUAD based on data from GEO, Oncomine, TCGA, and our in-house RT-qPCR. (A) Forest plot of 11 records evaluating NEAT1 expression in LUAD (fixed-effects model). If the SMD $>0$ and the corresponding 95\% CI of the SMD did not overlap zero, NEAT1 was upregulated in LUAD tissues compared with normal lung tissues. (B) Forest plot of 10 records evaluating miR-193a-3p expression in LUAD (random-effects model). If the $\mathrm{SMD}<0$ and the corresponding $95 \% \mathrm{CI}$ of the SMD did not overlap zero, miR-193a-3p was downregulated in LUAD tissues compared with normal lung tissues.

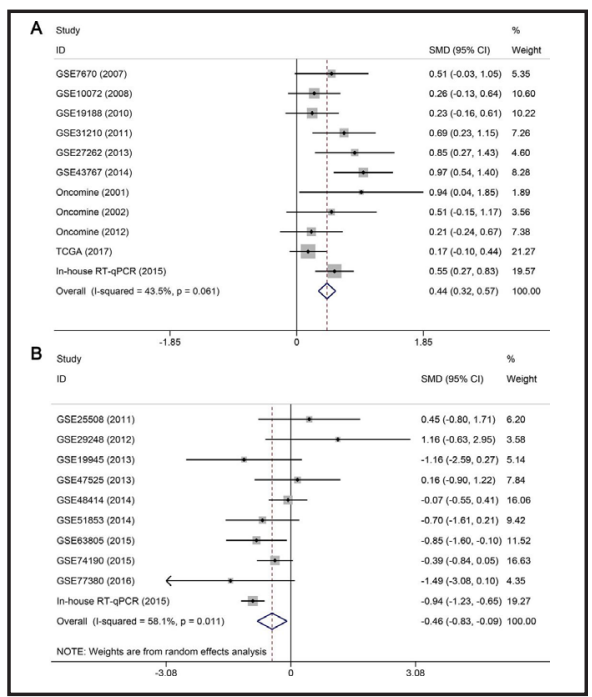

Fig. 11. Sensitivity analysis evaluating meta-analysis stability. (A) Sensitivity analysis indicated that the pooled SMD for NEAT1 was stable. (B) Sensitivity analysis indicated that the pooled SMD for miR-193a-3p was stable.

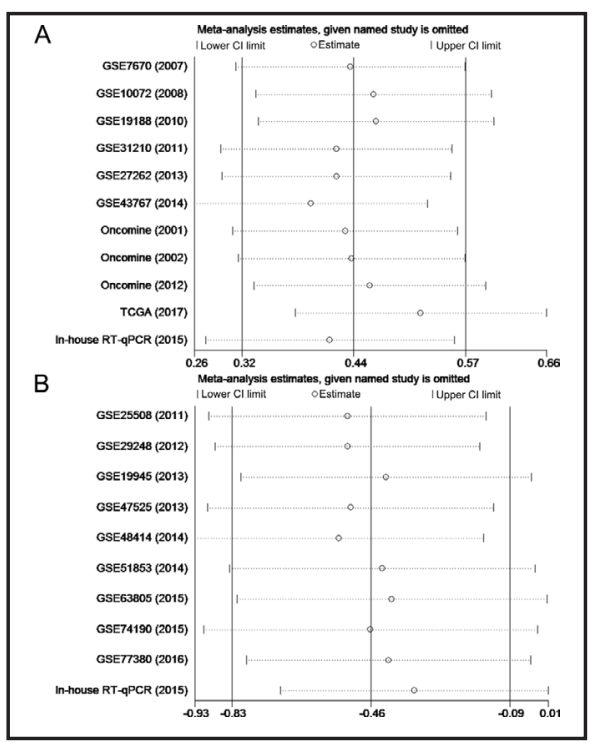


0.57, $\mathrm{P}<0.0001 ; \mathrm{I}^{2}=43.5 \%, \mathrm{P}=0.061 ;$ Fig. 10A). In contrast, the expression of miR193a-3p was lower in LUAD tissues than in normal lung tissues $(\mathrm{SMD}=-0.46,95 \% \mathrm{CI}=-$ 0.83 to $-0.09, \mathrm{P}=0.014 ; \mathrm{I}^{2}=58.1 \%, \mathrm{P}=0.011$; Fig. 10B). Sensitivity analyses suggested that the pooled results were stable (Fig. 11). Begg's and Egger's tests indicated no publication bias (NEAT1: Begg's $\mathrm{P}=0.213$, Egger's $\mathrm{P}=0.137$; Fig. 12A; miR-193a-3p: Begg's $\mathrm{P}=0.721$, Egger's $\mathrm{P}=0.281$; Fig. 12B).

Simultaneously, sROC curves were also generated to illustrate the ability of NEAT1 and miR-193a-3p expressions to differentiate LUAD patients from normal controls. As shown in Fig. 13, the area under the curve (AUC) for NEAT1 was 0.75 (95\% CI=0.71-0.78), with a sensitivity and specificity of $0.90(95 \% \mathrm{CI}=0.76-0.96)$ and 0.43 (95\% CI=0.25-0.63), respectively. As for miR-193a-3p, the AUC value was 0.77 (95\% CI=0.73-0.80), with a sensitivity and specificity of $0.85(95 \% \mathrm{CI}=0.61-0.95)$ and 0.61 (95\% CI=0.42-0.76), respectively.

NEAT1 may compete with USF1 for binding to miR-193a-3p as a ceRNA

Putative targets of miR-193a-3p were obtained from 12 prediction algorithms. A total of 285 genes that appeared in at least 8 algorithms were considered targets of miR-193a-3p. Simultaneously, we collected 552 genes positively correlated with NEAT1 from cBioPortal, a database that collects and processes data from TCGA. The intersection between the predicted target genes and positively correlated genes was determined (Fig. 14). In all, six overlapping genes (PIGA, USF1, RGS12, CLEC16A, MMP19, and KHNYN) were obtained for further analyses. The expression patterns of the six genes were visualized in the form of a heatmap and box-scatter plots based on data from TCGA (Fig. 15). According to our results, both USF1 and KHNYN were significantly upregulated in LUAD. We then predicted the binding sites of miR-193a-3p with USF1 and KHNYN and found that only the USF1 gene contained conserved miR-193a-3p binding sites (Fig. 16A). The correlation between USF1 and NEAT1 is shown in Fig. 16B. Furthermore, the overexpression of USF1 in LUAD was confirmed by data from Oncomine (Fig. 17).

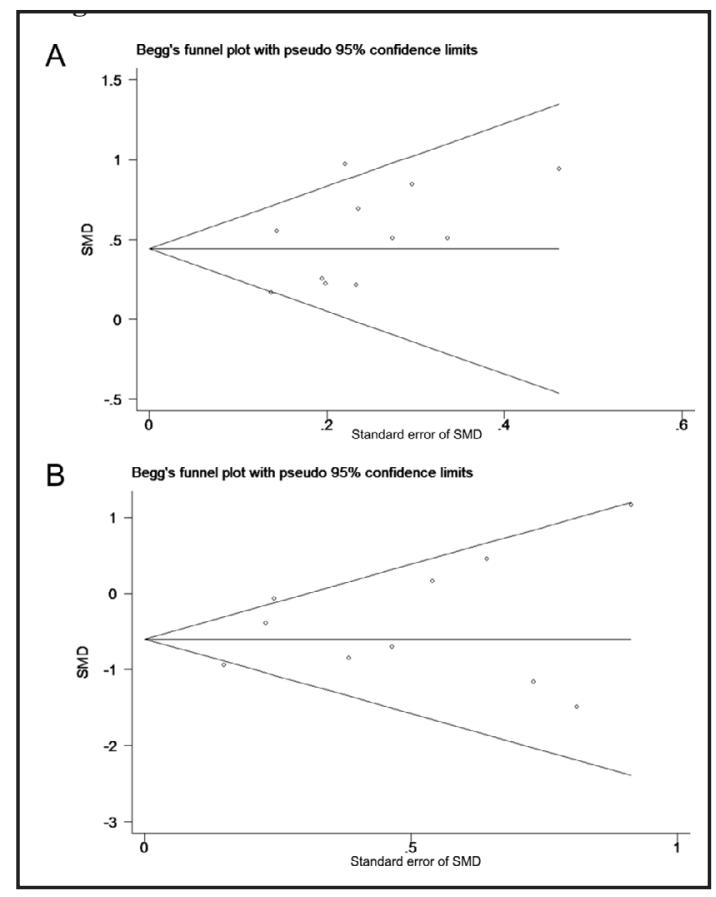

Fig. 12. Potential publication bias among included studies. (A) Funnel plot of 11 included studies for NEAT1. (B) Funnel plot of the 10 included studies for miR-193a-3p.

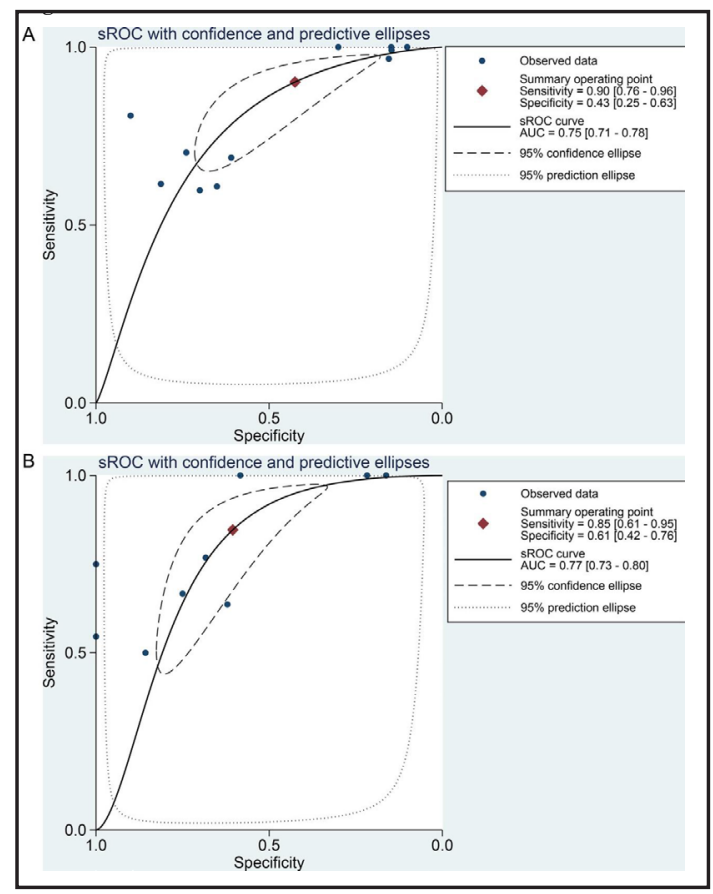

Fig. 13. sROC curves for the identification of LUAD patients from normal controls using NEAT1 and miR193a-3p expression. (A) Ability of NEAT1 expression to discriminate LUAD patients from normal controls. (B) Ability of miR-193a-3p expression to discriminate LUAD patients from normal controls. 
Fig. 14. Venn diagram of overlapping genes from the predicted target genes of miR-193a-3p and the positively correlated genes of NEAT1.

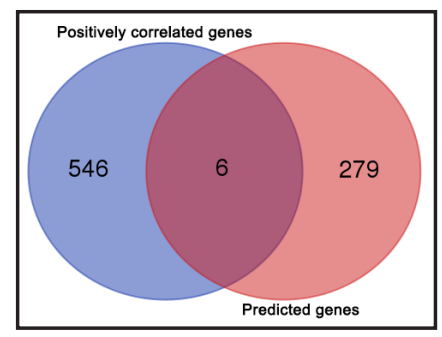

Fig. 15. Expression of the six overlapping genes (PIGA, USF1, RGS12, CLEC16A, MMP19, and KHNYN) in LUAD based on data from TCGA. (A) Heatmap of the six overlapping genes. (B) Box-scatter plots for PIGA, USF1, and RGS12 expression in LUAD. (C) Box-scatter plots for CLEC16A, MMP19, and KHNYN expression in LUAD.

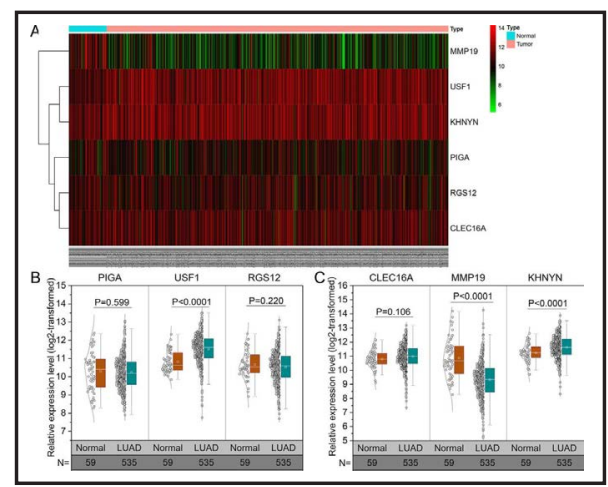

Fig. 16. Identification of USF1 as a potential target of miR193a-3p. (A) Predicted miR-193a-3p binding sites on USF1. (B) Correlation between USF1 and NEAT1 based on data from TCGA.

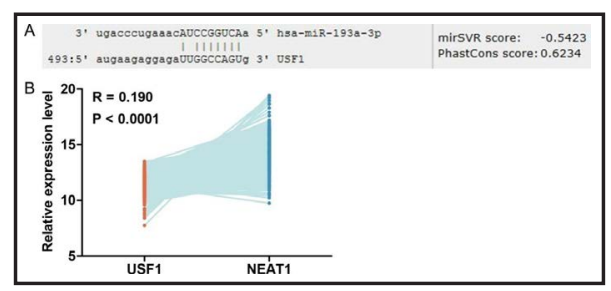

Fig. 17. Expression level of USF1 in LUAD based on data from Oncomine. (A) Study conducted by Semlamat SA. (B) Study conducted by Okayama H. (C) Study conducted by Beer DG.

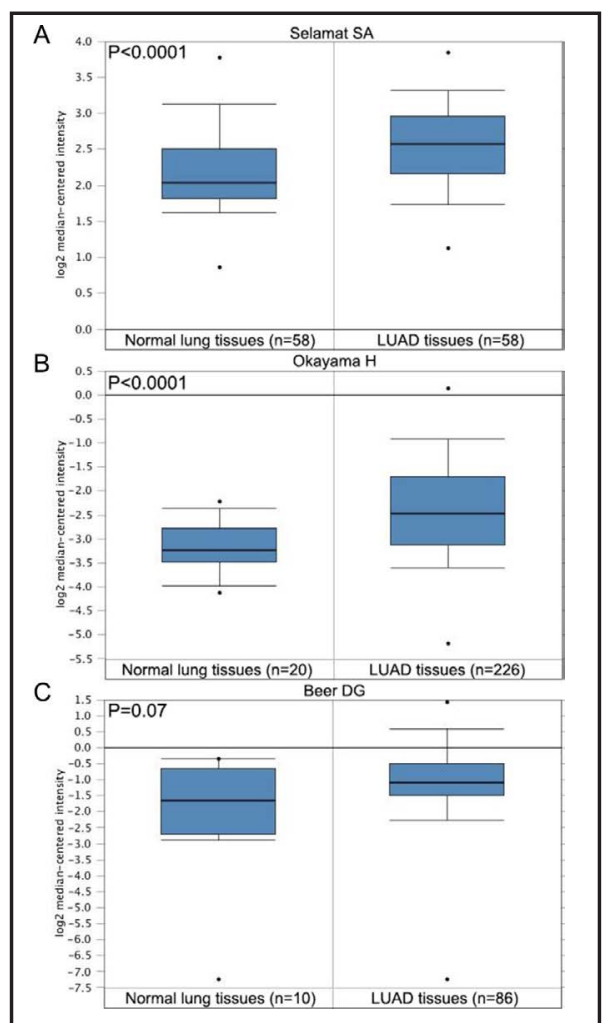




\section{Cellular Physiology Cell Physiol Biochem 2018;48:905-918 \begin{tabular}{l|l} 
DOI: 10.1159/000491958 & O 2018 The Author(s). Published by S. Karger AG, Basel \\
www.karger.com/cpb
\end{tabular}

Because USF1 is a protein-coding gene, we also validated the expression of its encoded protein using data from The Human Protein Atlas database (http://www.proteinatlas. org/). We found that protein USF1 was highly expressed in LUAD tissues compared with normal lung tissues (Fig. 18).

\section{Discussion}

Increasing studies have reported that lncRNAs, a class of RNA molecules with more than 200 nucleotides, perform complicated and critical roles in a myriad of malignant tumors at chromatin organizational, translational, and post-translational levels [23-27]. In-depth analysis of the exact actions and mechanisms of specific lncRNAs in tumorigenesis contributes to clinical treatment decision-making in patients with cancers.

The IncRNA NEAT1, an important

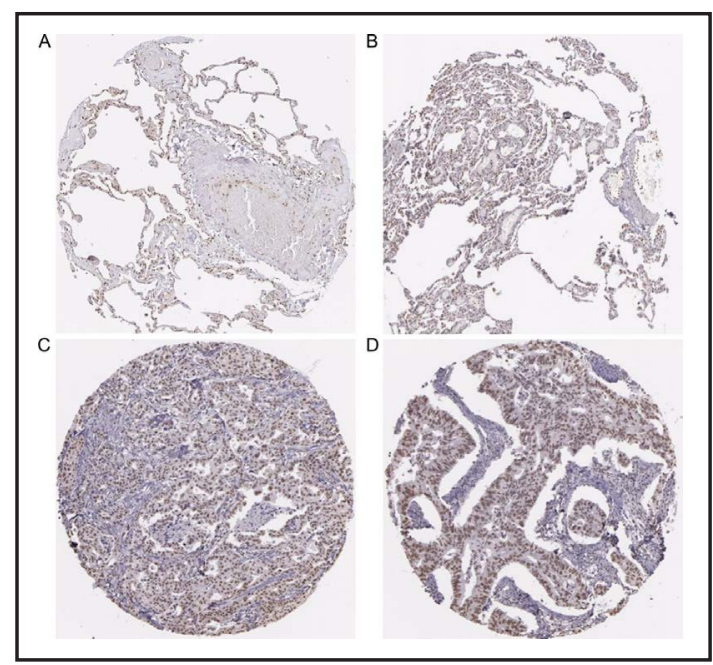

Fig. 18. Immunohistochemical staining of USF1 protein based on The Human Protein Atlas database (https://www.proteinatlas.org/). Paracancerous lung tissues showed moderate USF1 protein expression (A and B). LUAD tissues showed high USF1 protein expression (C and D) Magnification: $\times 100$. structural component of paraspeckle, regulates gene transcription and translation by retaining and editing mRNA in the cell nucleus [28-30]. Multiple reports have indicated that NEAT1 is involved in several malignant tumors, such as hepatocellular carcinoma [31], ovarian carcinoma [32], breast cancer [33], and endometrial carcinoma [34]. The close correlation between NEAT1 expression and NSCLC has also been investigated [11-14].

In this study, we aimed to uncover the biological function and underlying regulatory mechanism of NEAT1 in LUAD. First, we carried out in vitro experiments and found that knockdown of NEAT1 in LUAD cells markedly repressed cell proliferation, invasion, and migration and induced cell apoptosis, indicating that NEAT1 could drive LUAD development. However, one limitation should not be ignored in this study: different cell lines were used in different experiments, which may induce inhomogeneity in the results. Then, we focused on the potential molecular mechanism of NEAT1 in LUAD. Previous studies have demonstrated that NEAT1 promoted tumor development by functioning as a ceRNA or a molecular sponge to bind to miRNA and subsequently regulate cancer-related gene expression [35-38]. In this study, we probed the interaction between NEAT1 and miR-193a-3p. Bioinformatics analysis showed that NEAT1 harbored conserved miR-193a-3p cognate sites. Dual-luciferase reporter assay verified that miR-193a-3p directly targeted NEAT1 at its 3'-UTR. We further examined the expression of NEAT1 and miR-193a-3p in LUAD cells and normal lung epithelial cells. The results showed that NEAT1 was upregulated in LUAD cell lines (A549 and H1299) and miR-193a-3p was downregulated in LUAD cell lines (A549 and HCC827) compared with the normal lung cell line (BEAS-2B). However, the expression of NEAT1 in HCC827 cells was similar to that in BEAS-2B cells, and the expression of miR-193a-3p in H1299 cells was similar to that in BEAS-2B cells. Given the different genetic backgrounds of different cell lines, we conjecture that NEAT1 and miR-193a-3p are selectively expressed in different LUAD cell lines. Further study is necessary to corroborate these findings. Our research group has previously detected NEAT1 and miR-193a-3p expression in 125 NSCLC and adjacent non-tumorous lung samples by RT-qPCR, and we found high expression of NEAT1 and low expression of miR-193a-3p in NSCLC tissues [9, 39]. However, the expression levels of NEAT1 and miR-193a in LUAD tissues have not yet been separately elucidated. In this study, we combined the microarray and RNA-seq data from GEO, Oncomine, and TCGA and the in- 


\section{Cellular Physiology Cell Physiol Biochem 2018;48:905-918 \begin{tabular}{l|l} 
DOI: 10.1159/000491958 & O 2018 The Author(s). Published by S. Karger AG, Basel \\
www.karger.com/cpb
\end{tabular} Xiong et al.: NEAT1 Promotes Lung Adenocarcinoma Deterioration}

house RT-qPCR data from our previous studies to systematically investigate the expression levels of NEAT1 and miR-193a-3p in LUAD. The pooled results showed that NEAT1 was upregulated while miR-193a-3p was downregulated in LUAD tissues. All of these findings indicated a targeted regulation of NEAT1 and miR-193a-3p.

Numerous studies have demonstrated that miR-193a-3p plays vital roles in various cancers, including lung cancer. Yu et al [40]. detected miR-193a-3p expression in NSCLC and adjacent non-tumorous lung tissues. They found that miR193a-3p was downregulated in NSCLC tissues and that decreased miR-193a-3p was correlated

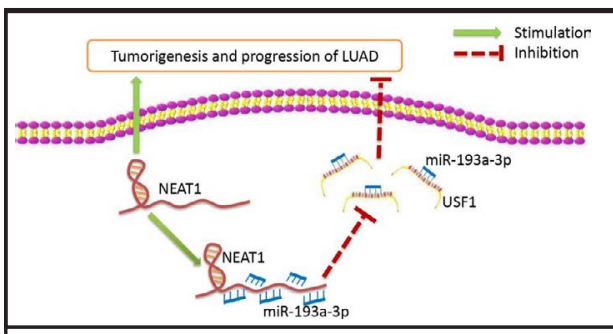

Fig. 19. Underlying regulatory mechanism of NEAT1 in LUAD. NEAT1 may exert its role by functioning as a ceRNA to sponge miR-193a$3 p$ and relieve the inhibitory effect of miR$193 a-3 p$ on the USE1 gene. with TNM stage as well as lymphatic metastasis. The authors also discovered that an enforced expression of miR-193a-3p restrained NSCLC metastasis in vitro and in vivo by negatively regulating the ERBB4/S6K2 signaling cascade. Similarly, Liang et al [41]. also demonstrated the target effect of miR-193a-3p on ERBB4 in NSCLC. A study carried out by Fan et al [42]. showed that miR-193a-3p has anti-tumor functions via negative modulation of its target KRAS. The interaction between miR-193a-3p and IncRNA has also been reported. A study conducted by Nie et al [43]. indicated that lncRNA UCA1 competitively sponged miR-193a-3p in NSCLC and thus increased the expression of ERBB4, a target of miR-193a-3p. In our study, we demonstrated that NEAT1 could directly bind to miR-193a-3p. To further explore whether NEAT1 acts as a ceRNA and antagonizes the inhibitory effect of miR-193a-3p on its target genes, we searched for putative target genes of miR-193a-3p in 12 online prediction databases and determined genes positively correlated with NEAT1 as candidate targets. Subsequently, we analyzed the expression of these selected genes with data from TCGA. The results showed that the USF1 gene was not only upregulated in LUAD, but also positively correlated with NEAT1. Hence, we hypothesized that NEAT1 may function as a ceRNA to sponge miR-193a-3p and relieve the inhibitory effect of miR-193a-3p on the USF1 gene. The potential regulatory mechanism is shown in Fig. 19. Further in vitro and in vivo experiments are indispensable to validate our findings.

In summary, our results indicate that an overexpressed NEAT1 facilitates the occurrence and progression of LUAD. NEAT1 may exert its role by functioning as a ceRNA to regulate miR193a-3p. These findings provide a new perspective on the underlying molecular mechanism of NEAT1 in LUAD, suggesting that NEAT1 could be a valuable biomarker and therapeutic target in patients with LUAD.

\section{Acknowledgements}

This study is supported by the National Natural Science Foundation of China (NSFC81760420, NSFC81560469 and NSFC 81360327), the National Natural Science Foundation of Guangxi, China (2017GXNSFAA198016, 2016GXNSFAA380255 and 2015GXNSFCA139009), the Guangxi Medical University Training Program for Distinguished Young Scholars (2017), the Medical Excellence Award Funded by the Creative Research Development Grant from the First Affiliated Hospital of Guangxi Medical University, and the Fund of Guangxi Provincial Health Bureau Scientific Research Project (Z2018).

\section{Disclosure Statement}

The authors declare to have no conflict of interests. 


\section{Cellular Physiology Cell Physiol Biochem 2018;48:905-918 \begin{tabular}{l|l} 
DOI: 10.1159/000491958 & $\begin{array}{l}\text { O 2018 The Author(s). Published by S. Karger AG, Basel } \\
\text { www.karger.com/cpb }\end{array}$ \\
\hline and Biochemistry
\end{tabular}}

Xiong et al.: NEAT1 Promotes Lung Adenocarcinoma Deterioration

\section{References}

1 Siegel RL, Miller KD, Jemal A: Cancer Statistics, 2017 CA Cancer J Clin 2017;67:7-30.

2 Chen W, Zheng R, Baade PD, Zhang S, Zeng H, Bray F, Jemal A, Yu XQ He J: Cancer statistics in China, 2015 CA Cancer J Clin 2016;66:115-132.

3 Wei S, Zhang ZY, Fu SL, Xie JG, Liu XS, Xu YJ, Zhao JP, Xiong WN: Hsa-miR-623 suppresses tumor progression in human lung adenocarcinoma. Cell Death Dis 2016;7:e2388.

4 Ali SA, Justilien V, Jamieson L, Murray NR, Fields AP: Protein Kinase Ciota Drives a NOTCH3-dependent Stem-like Phenotype in Mutant KRAS Lung Adenocarcinoma. Cancer Cell 2016;29:367-378.

5 Wang Y, He L, Du Y, Zhu P, Huang G, Luo J, Yan X, Ye B, Li C, Xia P, Zhang G, Tian Y, Chen R, Fan Z: The long noncoding RNA lncTCF7 promotes self-renewal of human liver cancer stem cells through activation of Wnt signaling. Cell Stem Cell 2015;16:413-425.

-6 Lin PC, Huang HD, Chang CC, Chang YS, Yen JC, Lee CC, Chang WH, Liu TC, Chang JG: Long noncoding RNA TUG1 is downregulated in non-small cell lung cancer and can regulate CELF1 on binding to PRC2 BMC Cancer 2016;16:583.

7 Terashima M, Tange S, Ishimura A, Suzuki T: MEG3 Long Noncoding RNA Contributes to the Epigenetic Regulation of Epithelial-Mesenchymal Transition in Lung Cancer Cell Lines. J Biol Chem 2017;292:82-99.

8 Guo F, Guo L, Li Y, Zhou Q, Li Z: MALAT1 is an oncogenic long non-coding RNA associated with tumor invasion in non-small cell lung cancer regulated by DNA methylation. Int J Clin Exp Pathol 2015;8:1590315910.

9 Pan LJ, Zhong TF, Tang RX, Li P, Dang YW, Huang SN, Chen G: Upregulation and clinicopathological significance of long non-coding NEAT1 RNA in NSCLC tissues. Asian Pac J Cancer Prev 2015;16:2851-2855.

10 Sun SJ, Lin Q, Ma JX, Shi WW, Yang B, Li F: Long non-coding RNA NEAT1 acts as oncogene in NSCLC by regulating the Wnt signaling pathway. Eur Rev Med Pharmacol Sci 2017;21:504-510.

11 Li S, Yang J, Xia Y, Fan Q Yang KP: LncRNA NEAT1 Promotes Proliferation and Invasion via Targeting MiR181a-5p in Non-Small Cell Lung Cancer. Oncol Res 2017;26:289-296.

12 Wu F, Mo Q, Wan X, Dan J, Hu H: NEAT1/has-mir-98-5p/MAPK6 axis is involved in non-small-cell lung cancer (NSCLC) development. J Cell Biochem 2017 Doi: 10.1002/jcb.26442.

-13 Sun C, Li S, Zhang F, Xi Y, Wang L, Bi Y, Li D: Long non-coding RNA NEAT1 promotes non-small cell lung cancer progression through regulation of miR-377-3p-E2F3 pathway. Oncotarget 2016;7:51784-51814.

14 Zhang J, Li Y, Dong M, Wu D: Long non-coding RNA NEAT1 regulates E2F3 expression by competitively binding to miR-377 in non-small cell lung cancer. Oncol Lett 2017;14:4983-4988.

15 Livak KJ, Schmittgen TD: Analysis of relative gene expression data using real-time quantitative PCR and the 2(-Delta Delta C(T)) Method. Methods 2001;25:402-408.

16 Chen G, Kronenberger P, Teugels E, De Greve J: Influence of RT-qPCR primer position on EGFR interference efficacy in lung cancer cells. Biol Proced Online 2010;13:1.

17 Chen G, Umelo IA, Lv S, Teugels E, Fostier K, Kronenberger P, Dewaele A, Sadones J, Geers C, De Greve J: miR-146a inhibits cell growth, cell migration and induces apoptosis in non-small cell lung cancer cells. PLoS One 2013;8:e60317.

18 Chen G, Kronenberger P, Teugels E, Umelo IA, De Greve J: Effect of siRNAs targeting the EGFR T790M mutation in a non-small cell lung cancer cell line resistant to EGFR tyrosine kinase inhibitors and combination with various agents. Biochem Biophys Res Commun 2013;431:623-629.

19 Chen G, Rong M, Luo D: TNFRSF6B neutralization antibody inhibits proliferation and induces apoptosis in hepatocellular carcinoma cell. Pathol Res Pract 2010;206:631-641.

20 Li S, Yue Y, Xu W, Xiong S: MicroRNA-146a represses mycobacteria-induced inflammatory response and facilitates bacterial replication via targeting IRAK-1 and TRAF-6PLoS One 2013;8:e81438.

21 Park H, Huang X, Lu C, Cairo MS, Zhou X: MicroRNA-146a and microRNA-146b regulate human dendritic cell apoptosis and cytokine production by targeting TRAF6 and IRAK1 proteins. J Biol Chem 2015;290:2831-2841.

22 Xiao F, Lan A, Lin Z, Song J, Zhang Y, Li J, Gu K, Lv B, Zhao D, Zeng S, Zhang R, Zhao W, Pan Z, Deng X, Yang $\mathrm{X}$ : Impact of CAG repeat length in the androgen receptor gene on male infertility - a meta-analysis. Reprod Biomed Online 2016;33:39-49.

23 Yang G, Lu X, Yuan L: LncRNA: a link between RNA and cancer. Biochim Biophys Acta 2014;1839:10971109. 


\section{Cellular Physiology Cell Physiol Biochem 2018;48:905-918 \begin{tabular}{l|l} 
and Biochemistry Published online: July 23, 2018 & $\begin{array}{l}\text { D) } 2018 \text { The Author(s). Published by S. Karger AG, Basel } \\
\text { www.karger.com/cpb }\end{array}$ \\
\hline
\end{tabular}}

24 Mendell JT: Targeting a Long Noncoding RNA in Breast Cancer. N Engl J Med 2016;374:2287-2289.

25 Zhou X, Chen H, Zhu L, Hao B, Zhang W, Hua J, Gu H, Jin W, Zhang G: Helicobacter pylori infection related long noncoding RNA (lncRNA) AF147447 inhibits gastric cancer proliferation and invasion by targeting MUC2 and up-regulating miR-34c. Oncotarget 2016;7:82770-82782.

-26 Cheng Z, Guo J, Chen L, Luo N, Yang W, Qu X: A long noncoding RNA AB073614 promotes tumorigenesis and predicts poor prognosis in ovarian cancer. Oncotarget 2015;6:25381-25389.

-27 Eoh KJ, Paek J, Kim SW, Kim HJ, Lee HY, Lee SK, Kim YT: Long non-coding RNA, steroid receptor RNA activator (SRA), induces tumor proliferation and invasion through the NOTCH pathway in cervical cancer cell lines. Oncol Rep 2017;38:3481-3488.

-28 Adriaens C, Marine JC: NEAT1-containing paraspeckles: Central hubs in stress response and tumor formation. Cell Cycle 2017;16:137-138.

29 Kawaguchi T, Hirose T: Chromatin remodeling complexes in the assembly of long noncoding RNAdependent nuclear bodies. Nucleus 2015;6:462-467.

30 Clemson CM, Hutchinson JN, Sara SA, Ensminger AW, Fox AH, Chess A, Lawrence JB: An architectural role for a nuclear noncoding RNA: NEAT1 RNA is essential for the structure of paraspeckles. Mol Cell 2009;33:717-726.

-31 Zheng X, Zhang Y, Liu Y, Fang L, Li L, Sun J, Pan Z, Xin W, Huang P: HIF-2alpha activated lncRNA NEAT1 promotes hepatocellular carcinoma cell invasion and metastasis by affecting the epithelial-mesenchymal transition. J Cell Biochem 2017;119:3247-3256.

-32 Ding N, Wu H, Tao T, Peng E: NEAT1 regulates cell proliferation and apoptosis of ovarian cancer by miR34a-5p/BCL2 Onco Targets Ther 2017;10:4905-4915.

-33 Li X, Wang S, Li Z, Long X, Guo Z, Zhang G, Zu J, Chen Y, Wen L: The IncRNA NEAT1 facilitates cell growth and invasion via the miR-211/HMGA2 axis in breast cancer. Int J Biol Macromol 2017;105:346-353.

-34 Wang J, Zhao X, Guo Z, Ma X, Song Y, Guo Y: Regulation of NEAT1/miR-214-3p on the growth, migration and invasion of endometrial carcinoma cells. Arch Gynecol Obstet 2017;295:1469-1475.

-35 Zhang XN, Wang CC, Zhou J: The long noncoding RNA NEAT1 contributes to hepatocellular carcinoma development by sponging miR-485 and enhancing the expression of the STAT3. J Cell Physiol 2017 Doi: $10.1002 /$ jcp.26371.

-36 Liu F, Chen N, Gong Y, Xiao R, Wang W, Pan Z: The long non-coding RNA NEAT1 enhances epithelialto-mesenchymal transition and chemoresistance via the miR-34a/c-Met axis in renal cell carcinoma. Oncotarget 2017;8:62927-62938.

-37 Qian K, Liu G, Tang Z, Hu Y, Fang Y, Chen Z, Xu X: The long non-coding RNA NEAT1 interacted with miR-101 modulates breast cancer growth by targeting EZH2 Arch Biochem Biophys 2017;615:1-9.

-38 Wang Y, Wang C, Chen C, Wu F, Shen P, Zhang P, He G, Li X: Long non-coding RNA NEAT1 regulates epithelial membrane protein 2 expression to repress nasopharyngeal carcinoma migration and irradiation-resistance through miR-101-3p as a competing endogenous RNA mechanism. Oncotarget 2017;8:70156-70171.

-39 Ren F, Ding H, Huang S, Wang H, Wu M, Luo D, Dang Y, Yang L, Chen G: Expression and clinicopathological significance of miR-193a-3p and its potential target astrocyte elevated gene-1 in non-small lung cancer tissues. Cancer Cell Int 2015;15:80.

40 Yu T, Li J, Yan M, Liu L, Lin H, Zhao F, Sun L, Zhang Y, Cui Y, Zhang F, Li J, He X, Yao M: MicroRNA-193a-3p and -5p suppress the metastasis of human non-small-cell lung cancer by downregulating the ERBB4/PIK3R3/ mTOR/S6K2 signaling pathway. Oncogene 2015;34:413-423.

41 Liang H, Liu M, Yan X, Zhou Y, Wang W, Wang X, Fu Z, Wang N, Zhang S, Wang Y, Zen K, Zhang CY, Hou D, Li J, Chen X: miR-193a-3p functions as a tumor suppressor in lung cancer by down-regulating ERBB4. J Biol Chem 2015;290:926-940.

42 Fan Q, Hu X, Zhang H, Wang S, Zhang H, You C, Zhang CY, Liang H, Chen X, Ba Y: MiR-193a-3p is an Important Tumour Suppressor in Lung Cancer and Directly Targets KRAS. Cell Physiol Biochem 2017;44:1311-1324.

43 Nie W, Ge HJ, Yang XQ Sun X, Huang H, Tao X, Chen WS, Li B: LncRNA-UCA1 exerts oncogenic functions in non-small cell lung cancer by targeting miR-193a-3p. Cancer Lett 2016;371:99-106. 